Supporting Information for:

\title{
Morphogenesis of the Evaporation-Induced Self-Assemblies of Polypyrrole Nanoparticles Dispersed in a Liquid Medium
}

\author{
Jyongsik Jang* and Joon Hak Oh \\ Hyperstructured Organic Materials Research Center and School of Chemical Engineering, \\ Seoul National University, Shinlimdong 56-1, Seoul 151-742, Korea
}

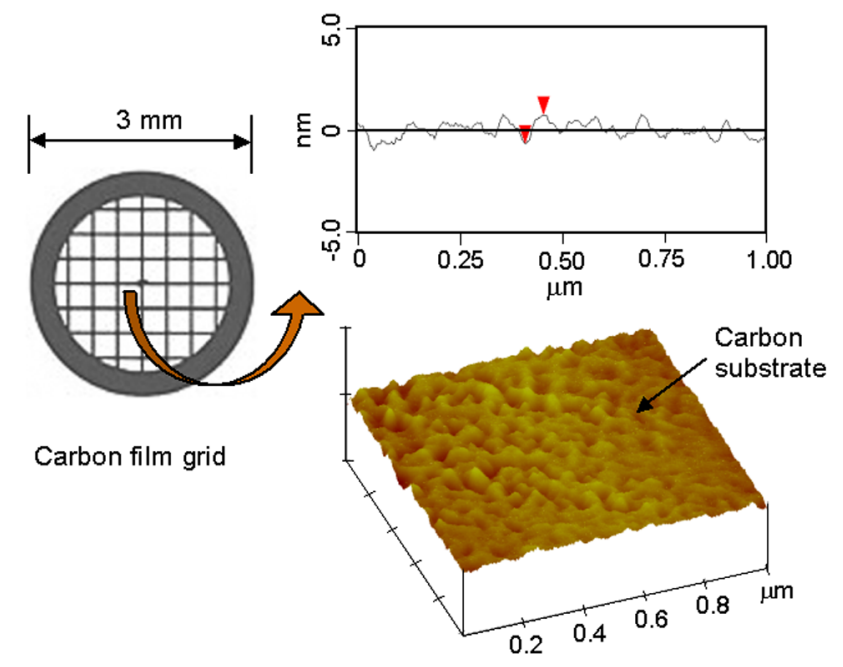

Figure S1. Carbon film grid and the AFM image (tapping mode) of film surface. The root-mean squared surface roughness of the carbon substrate was $0.34 \mathrm{~nm}$. 
(a)
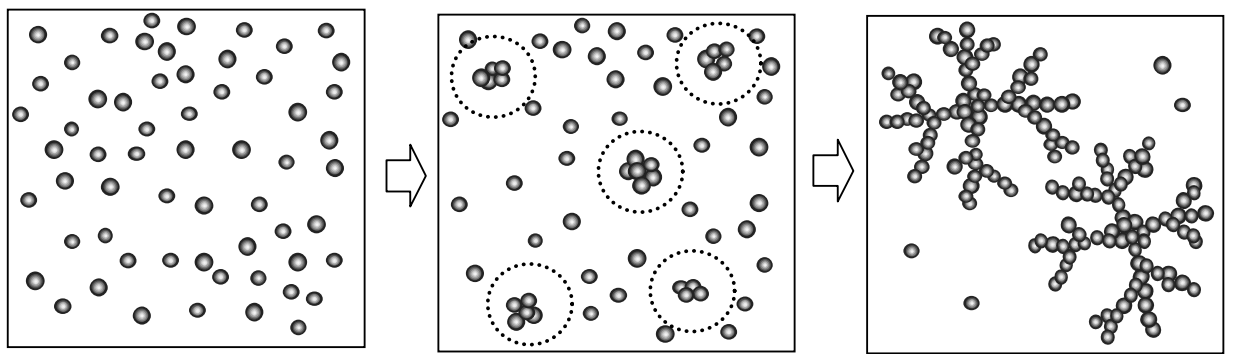

(b)

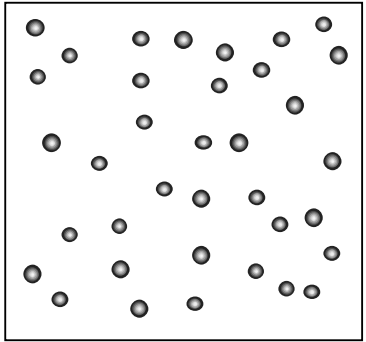

PPy nanoparticles dispersed in a liquid medium

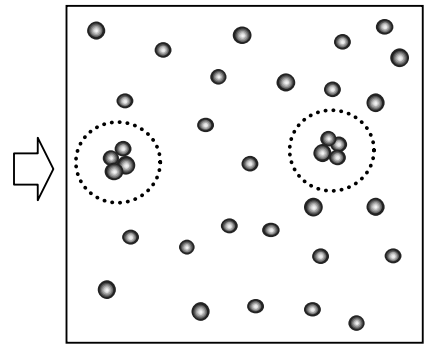

Nucleation

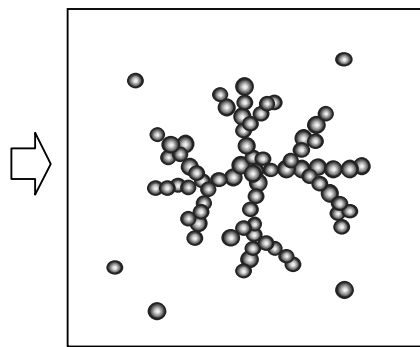

2-D dendrites

Figure S2. Schematic illustration of dendritic growth on a carbon support film at a high evaporation rate of the solvent: (a) high nanoparticle concentration, and (b) low nanoparticle concentration.

(a)

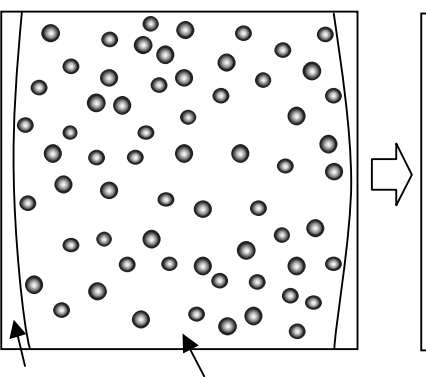

Carbon mesh Liquid

(b)

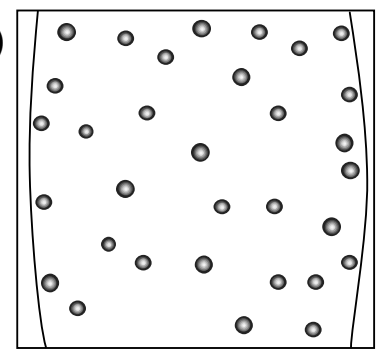

PPy nanoparticles dispersed in a liquid medium
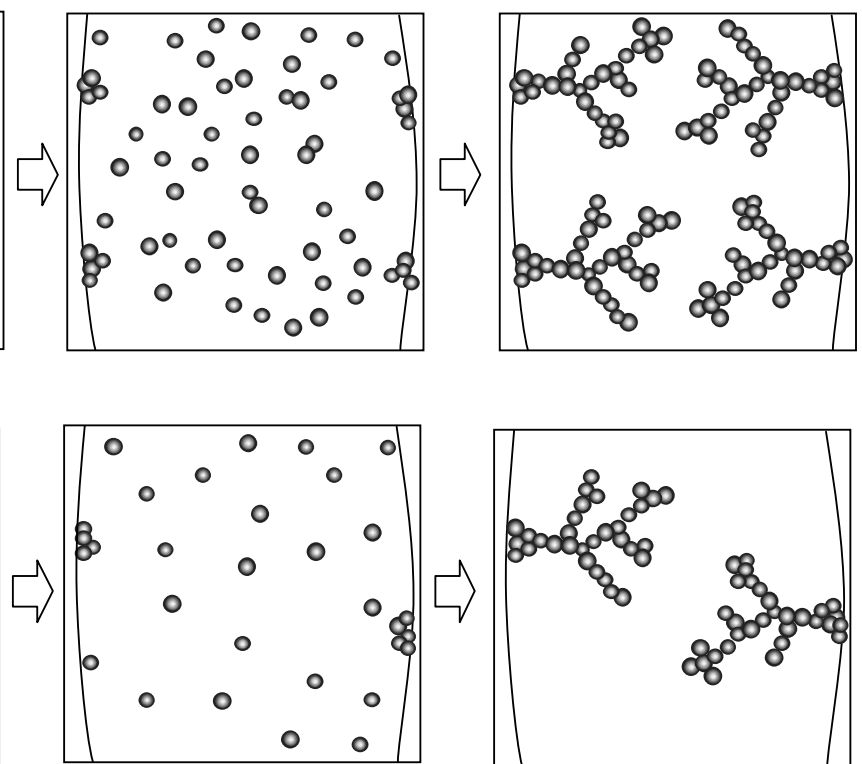

Nucleation

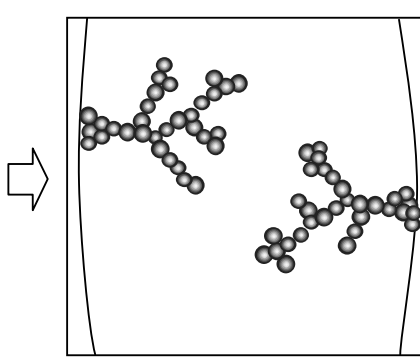

Pseudo 1-D dendrites

Figure S3. Schematic explanation of dendritic growth on a carbon net-mesh grid at a high evaporation rate of the solvent: (a) high nanoparticle concentration, and (b) low nanoparticle concentration. 
(a)

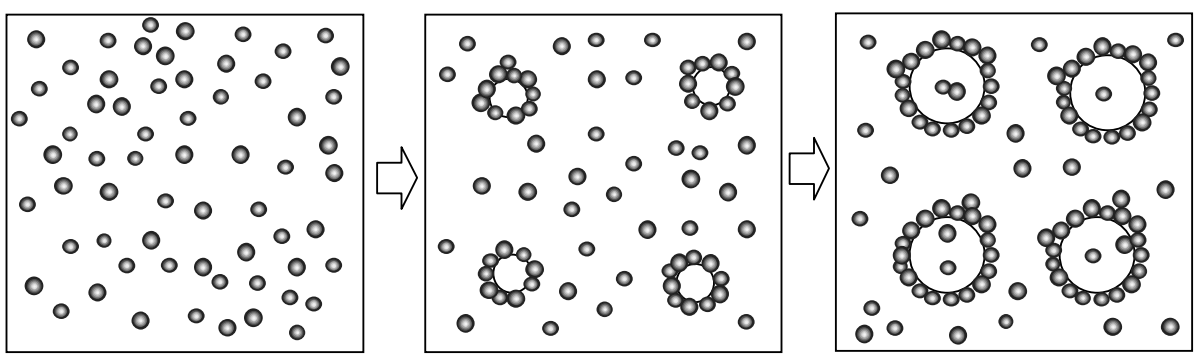

(b)
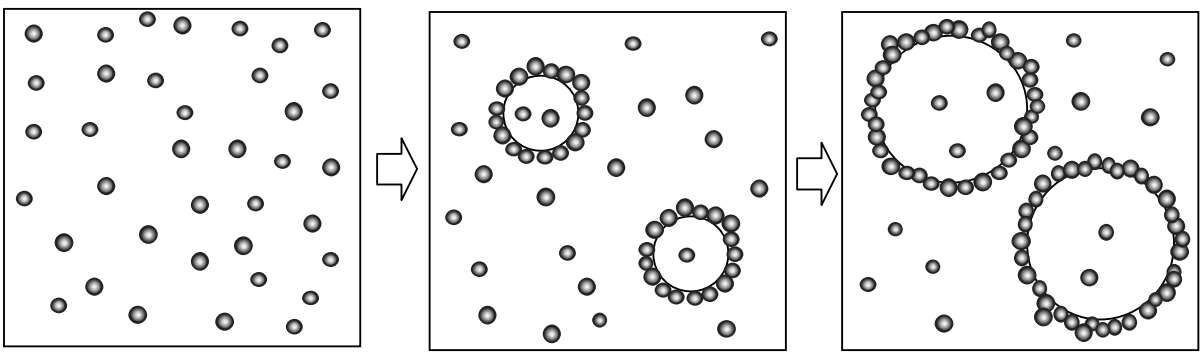

(c)

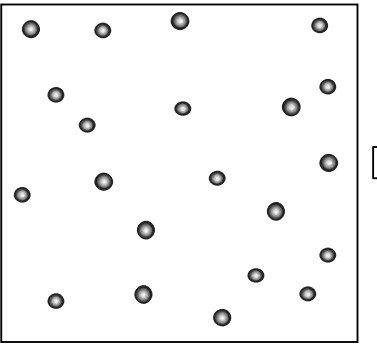

PPy nanoparticles dispersed in a liquid medium

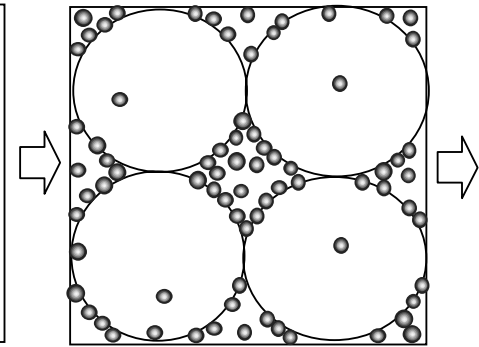

Hole opening

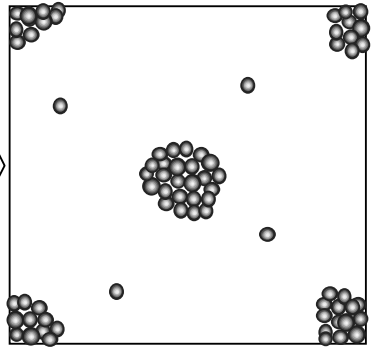

Pinned EISA morphology

Figure S4. Schematic representation for the formation mechanism of ring and spherulitic compact structures on a carbon net-mesh grid at a low evaporation rate of the solvent: (a) small ring, concentrated solution, (b) large ring, intermediate concentration, and (c) spherulitic compact assembly, dilute solution. The hole opening in slowly evaporating wet films pushes most PPy nanoparticles out along its advancing rim. The PPy nanoparticles in a concentrated solution can be more easily pinned, resulting in the formation of a smaller ring (a), compared with the case of a intermediate concentration (b). In the case of very low nanoparticle concentration, the growing hole are not pinned until they have run into one another, resulting in a closely packed aggregate (c). 


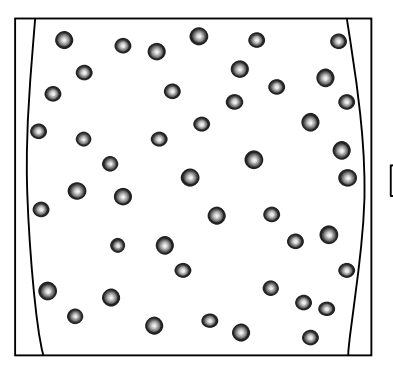

PPy nanoparticles dispersed in a liquid medium

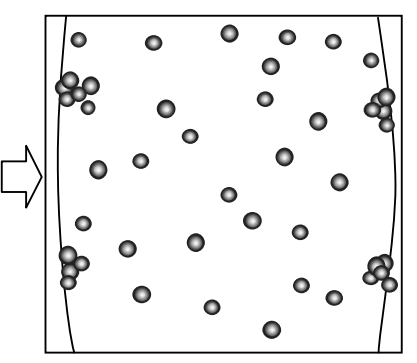

Nucleation

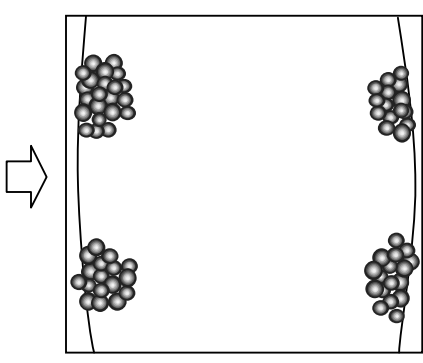

Closely packed assemblies on the carbon mesh

Figure S5. Schematic illustration of closely packed aggregates formed on a carbon net-mesh grid at a low evaporation rate of the solvent. Irrespective of the nanoparticle concentration, compact assemblies were only formed. The formation fraction of the closely packed assemblies increased with increasing the nanoparticle concentration due to the enhanced nucleation.

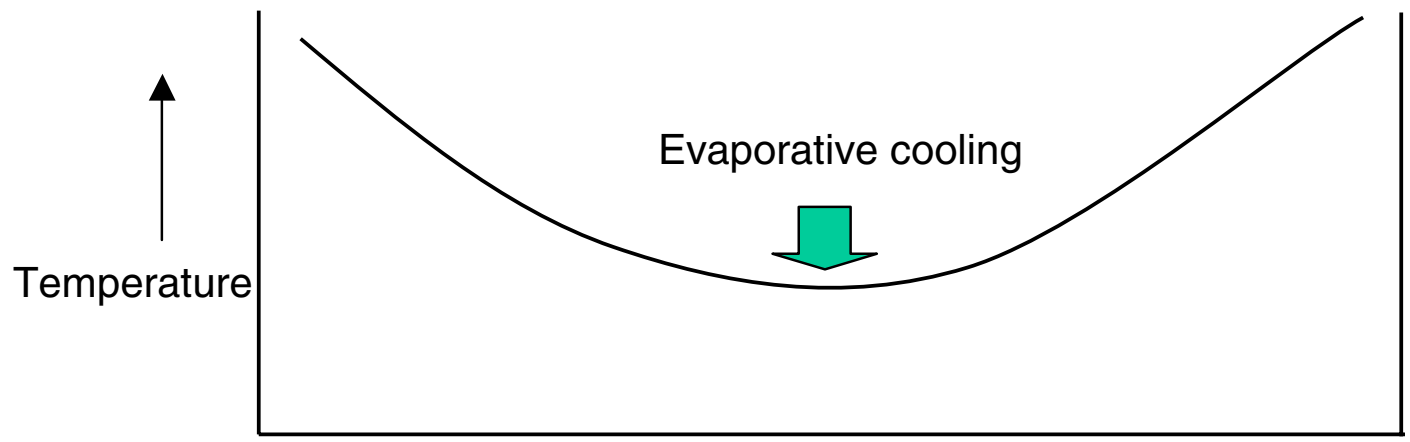

Evaporation

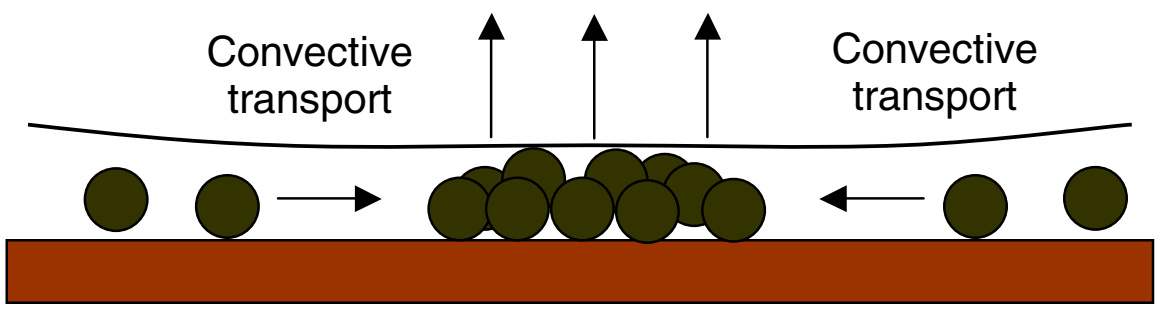

Figure S6. The schematic drawing of the temperature gradients due to evaporative cooling. The formation of nucleus causes the local imbalance of liquid film height. The liquid film spread on a core aggregate has a relatively thinner thickness and evaporates more rapidly. The difference in the evaporation rate causes the temperature gradients due to the evaporative cooling. 


\section{Surface coverage and of EISAs \& the average polydispersity in assembly size}

The surface coverage of EISAs was analyzed by averaging the area fraction of EISAs on the TEM images of the different 10 copper meshes in the same grid. The average size of EISAs was obtained by averaging those of 50 EISAs. In addition, the average nanoparticle size was also calculated by counting 50 nanoparticles. In these processes, we electronically scanned the TEM image and marked the contours of EISAs (for ring patterns, outer lines were marked and treated as solid spheres), and finally obtained the area fraction by evaluating the ratio of the selected pixel area to the overall pixel area. For the analysis of average diameter, the pixel length of the nanoparticle diameter was compared with that of scale bar. The average polydispersity of EISAs was as follows (50 EISAs considered):

2-D dendrites: $630 \pm 180 \mathrm{~nm}$

Pseudo 1-D dendrites: $380 \pm 50 \mathrm{~nm}$

Small ring patterns: $50 \pm 15 \mathrm{~nm}$

Large ring patterns: $180 \pm 55 \mathrm{~nm}$

Spherulitic compact structures: $210 \pm 70 \mathrm{~nm}$

Closely packed aggregates: $270 \pm 80 \mathrm{~nm}$ 